\title{
Design and simulation of bio fluidic sensor based on photonic crystal
}

\author{
G. K. Rajini*, A. Sharmila, V. Sankardoss \\ School of Electrical Engineering, VIT University, Vellore, India \\ *Corresponding author E-mail: rajini.gk@vit.ac.in
}

Copyright $\odot 2014$ G. K. Rajini et al. This is an open access article distributed under the Creative Commons Attribution License, which permits unrestricted use, distribution, and reproduction in any medium, provided the original work is properly cited.

\begin{abstract}
Photonic crystals are materials patterned with a periodicity in dielectric constant in one, two and three dimensions and associated with Bragg scattering which can create range of forbidden frequencies called Photonic Band Gap (PBG). By optimizing various parameters and creating defects, we will review the design and characterization of waveguides, optical cavities and multi-fluidic channel devices. We have used such waveguides and laser nanocavities as Biosensor, in which field intensity is strongly dependent on the type of biofliud and its refractive index. This design and simulation technique leads to development of a nanophotonic sensor for detection of biofluids. In this paper, we have simulated sensing of biofliud in various photonic defect structures with the help of a numerical algorithm called Finite Difference Time Domain (FDTD) method. The simulation result shows the high sensitivity for the change in the bio-molecular structure. For developing the complete sensor system, we have to use the MEMS technologies to integrate on-chip fluidic transport components with sensing systems. The resulting biofluidic system will have the capability to continuously monitor the concentration of a large number of relevant biological molecules continuously from ambulatory patients.
\end{abstract}

Keywords: FDTD, Photonic Crystals, Bio fluid Sensor, Optical Cavity.

\section{Introduction}

Photonic crystals are new classes of materials with which the properties of light can be controlled. These are regular array of materials with different refractive indices. Using such materials we can have complete control of microwave propagation. A metallic cavity does not allow electromagnetic waves to propagate below a certain threshold frequency and a metallic component which makes this method of optical control impossible to generalize. Photonic crystals not only mimic the properties of cavities and waveguides, but also applicable to wider range of frequencies. Analogous to the energy gap in pure semiconductor crystals, in which electrons are forbidden, these Photonic Band Gap (PBG) structures have a frequency stop band over which there is no transmission of electromagnetic waves [1] [2]. Similar to donor or acceptor state in a doped semiconductor a small defect introduced into the photonic crystals creates a resonant mode at a frequency that lies inside the band gap. These modes are called localized defect modes.

The photonic band gaps are created because of variation in the refractive indices. Higher the constant between the refractive indices, larger will be the photonic band gap. The simulation of the defect modes in photonic crystal was done in OptiFDTD software. Taking advantage of the low group velocity and certain mode distribution for some k-points in the band structure of a photonic crystal should enable the realization of very compact biosensor devices. Photonic crystals and optical band gap structures are facilitating the high precision control of electromagnetic field propagation, which are gaining ever increasing attention in both scientific and commercial applications. 


\section{Photonic crystal}

Photonic crystals are materials patterned with a periodicity in dielectric constant and associated with Bragg scattering which can create range of forbidden frequencies called Photonic Band Gap. In particular, lattice might introduce gaps into the energy band structure of the crystal, so that (due to Bragg-like diffraction from the atoms) electrons are forbidden to propagate with certain energies in certain directions. If the lattice potential is strong enough, the gap might extend to all possible directions, resulting in a complete band gap. If the dielectric constants of the materials are different enough and absorption of the material is minimum then scattering at the interfaces can produce many of the phenomenon as the atomic potential does for electrons. A metallic cavity does not allow electromagnetic waves to propagate below a threshold frequency and a metallic waveguide only allows propagation along its axis. These properties are useful at frequencies outside the microwave regime. The photonic structures are fabricated by holographic lithography technique [3].

The periodic variations of the dielectric constant in photonic crystals gives rise to a photonic band structure and produce many similar phenomena for photons as for the electrons in a semiconductor crystal. Light at energies within the PBG is prevented from propagating in certain directions in the structure, depending on the periodicity of the photonic crystal that can be of one, two or three dimensions.

\subsection{Important parameters of photonic crystals}

\subsubsection{Dimensionality}

The periodicity of the refractive index will determine the dimensionality of the photonic crystal. Example: one, two, three dimensional lattices are Bragg grating, cylindrical rods in air or holes in substrate and Woodpile structure respectively [1].

\subsubsection{Lattice parameter}

This fundamental distance is used to characterize the separation between scattering building blocks. In case of square lattice, it usually takes the side of the square. The range of wavelengths of the optical spectrum, where the photonic crystal works will be proportional to the lattice parameter.

\subsubsection{Filling fraction}

The relative amount of material composing the scattering building block is called filling fraction.

\subsubsection{Effective refractive index}

It is defined as the square root of the average dielectric constant. Similar to the lattice parameter, the wavelength range of the optical spectrum, where the photonic crystal works will depend on this parameter.

\subsubsection{Refractive index (RI) contrast}

It is defined as the ratio between RI of high dielectric constant material $n_{h}$ to the RI of low dielectric constant material $\mathrm{n}_{\mathrm{l}}$. This value offers a general idea of the scattering strength of photonic crystal. The photonic crystal benefits are small size and high integration density which facilitates the fabrication of waveguide bends, couplers, and interferometers are usually one hundred to one thousand times smaller than the commercially available.

\section{CPBG structures and FDTD technique}

The modulation in refractive index causes certain energies and direction forbidden for photons. A region of energies where the PC doesn't allow photons to propagate regardless of their direction and polarization is called Complete Photonic Band Gap (CPBG) structures [12]. It gives us information about propagation properties of electromagnetic radiation within the photonic crystal. The fig. 1 shows the photons propagating in the most important directions of the first Brillouin zone are the solution and collection is called Photonic Band Gap (PBG) structure. 

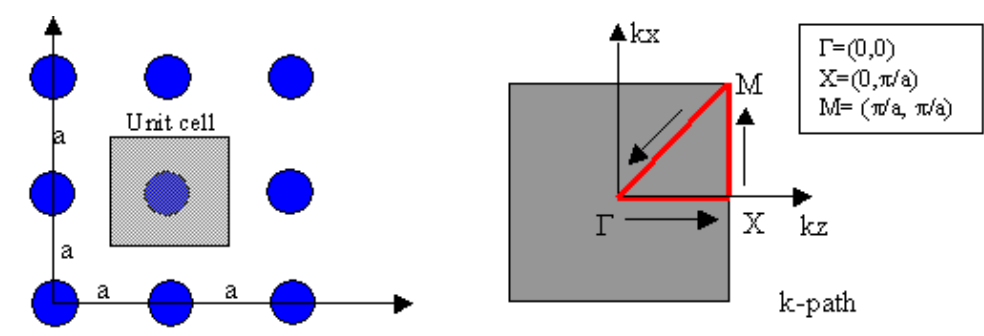

Fig. 1: Unit cell and associated Brillouin zone for square lattice

Brillouin Zone contains redundant copies of the irreducible zone. X, M is the vertices of irreducible zone. Other points in this Brillouin Zone can be obtained by applying symmetry operations like mirror reflection, inversion, rotation on the Brillouin Zone. The PBG effect was proposed in 1987 by Yablonovitch and John, and the subject has received an increasing interest since then [4]. One of the most interesting applications of photonic band gap materials are when constructing photonic band gap waveguides with properties different from conventional waveguides.

The Finite Difference Time Domain (FDTD) technique avoids the difficulties of linear algebra that limits the size of frequency domain integral equations and finite element electromagnetic models with as many as 109 field unknowns. It is accurate and robust. The sources of error in FDTD calculation are well understood and can be bounded to permit accurate models for a variety of electromagnetic wave interactions. Being a time-domain technique, it calculates impulse response of electromagnetic system; it also directly calculates the non-linear response.

An FDTD method which generates time marched arrays of field quantities suitable for use in color video's to illustrate field dynamics. FDTD related space grid time domain techniques are direct solution methods for Maxwell's equations [9]. These are based on volumetric sampling of unknown electric and magnetic fields within surrounding structure of interest. FDTD and related techniques are marching-in-time procedures that simulate the continuous actual electromagnetic waves in a finite spatial region of sampled data numerical analogs propagating in computer data spaces. Phenomenon such as induction of surface currents [5], scattering, multiple scattering, aperture penetration, cavity excitation are modeled time step by time step by the action of numerical analog to curl equations.

\section{Applications of photonic crystal}

Photonic crystal materials consist of periodic variations in the dielectric constant. This variation can be artificially created by etching holes into a uniform dielectric material. In the fabrication of integrated circuits using photonic crystals, where the information carriers are photons instead of electrons which provide us the most promising applications. The resulting photonic crystal will have optical properties that differ greatly from the original material. For example, one can create a nearly perfect mirror for some optical wavelengths by etching holes into silicon or indium phosphide [6]. Devices such as waveguides, couplers/splitter, resonators and prisms can be made by changing the size of few holes in the periodic photonic crystals fabric. By carefully utilizing their inherent wavelength dispersive properties, photonic crystal devices can be used in conjunction with more conventional refractive index guiding devices to build compact photonic integrated circuits for use in optical interconnects or optical sensors [10].

\subsection{Defects in photonic crystal}

Due to photonic band gap, most of the incident light is reflected but some of the photons can reach the defect. Once photons enter the defect, they are unable to escape the defect because of surrounding media, which reflect the photons back into the defect. As a consequence, light with a certain wavelength is trapped and accumulated around the defect in the photonic band gap structure. Most of the applications of photonic crystals discussed in the following sections utilize the localization of photons by defects. A defect creates allowed states for particular photon frequencies in the bandgap. The defects are two types namely point defect and line defect.

\subsubsection{Point defect}

A point defect within the photonic crystal could act as a microcavity that would trap light at a point. By removing a rod from the lattice, we can effectively create a cavity which is surrounded by reflecting walls. The resonant microcavities are integrated directly along the plane of the substrate which gives rise to strong field confinement and allow the efficient coupling into channel waveguides. By concentrating the field fluctuations into a small volume, the recombination rate of carriers will be increased. This could lead to the enhancement of spontaneous emission and allows the microcavities to be modulated at very high speeds. In a photonic crystal, sharp resonant states can be created in the vicinity of the defect. 


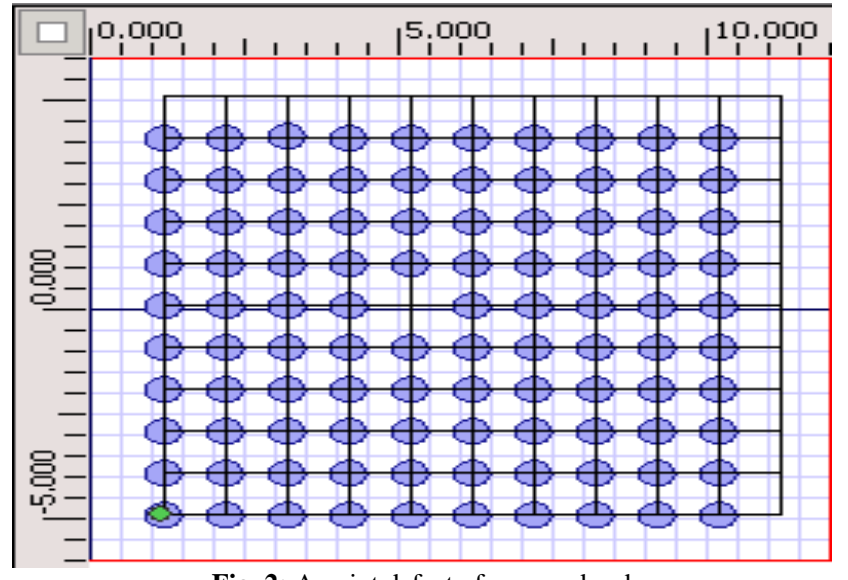

Fig. 2: A point defect of removed rod

The point defects can be produced by removing a single rod using Opti-FDTD software as shown in fig. 2. Cavity structures in PhCs are created by the removal of holes by introducing point defects into the crystal. A cavity waveguide consists of a point defect forming cavity in a PhC structure. The cavities are designed in order to have their resonant frequency inside the PBG of the surrounding crystal. The spacing between the adjacent defects is chosen to allow some overlap of the wave functions, although the resonant modes are tightly confined to the respective cavity. Light then propagates between adjacent cavities due to the electromagnetic field interaction, the so-called "Photon hopping". This is the classical optical wave analogue of the tight-binding approximation in solid state physics [7].

\subsubsection{Line defect}

A line defect within a photonic crystal can guide photons from one location to the other and it acts as waveguide. If the frequency of light lies within the CPBG, it is confined to the defect line, since propagation is forbidden through the rest of the photonic crystal. The main advantages of such waveguides are sharp bends does not present any transmission losses and $98 \%$ efficiency can be expected.

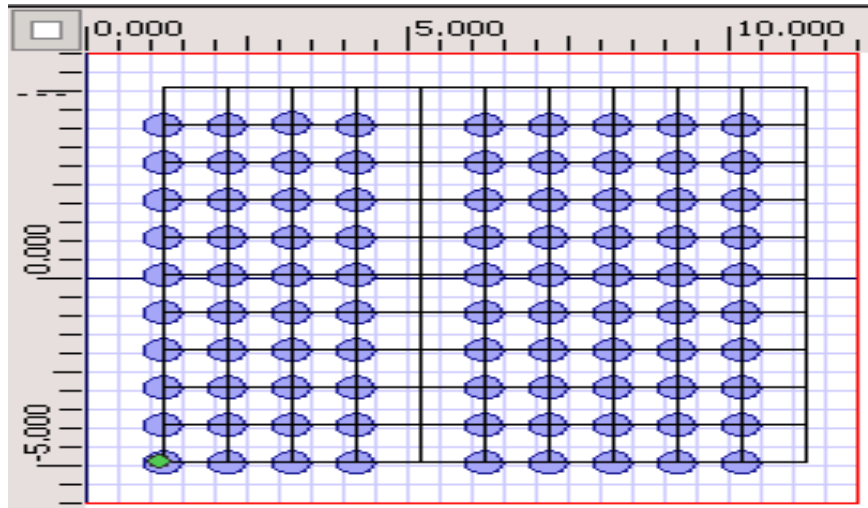

Fig. 3: A line defect of removed column of rods

The line defects can be produced by removing columns or rows of rods using Opti-FDTD software as shown in fig. 3. These defects can be created by removal of either column or row of high-index material. Generally altering the effective index of the waveguides, which operate on the principle of index guiding, necessarily confine light to regions of higher index. In contrast, photonic-crystal waveguides can guide light in regions of lower index, even primarily in air.

\section{Design of biofluidic sensor}

Blood plasma is a component of blood. It is the liquid in which blood cells are suspended. It contains proteins, nutrients, metabolic end products, hormones, and inorganic electrolytes. It is the storage and transport medium of clotting factors and its protein content is necessary to maintain the pressure of the blood. Biofluidic sensors are designed with three different structures using point and line defects. This paper presents some sensors which are designed using the Opti-FDTD software. These are as follows

a) A resonant cavity.

b) $\quad$ A $90^{\circ}$ bend waveguide.

c) A multifluidic channel. 


\subsection{A resonant cavity}

By creating point defect in photonic crystal a resonant cavity can be designed, such resonant cavities are crucial components of laser systems. A defect mode in photonic crystal would serve as an efficient resonant cavity, since it would only trap light in very narrow frequency band and would hardly suffer any losses. If the biofluid is placed in center of the cavity, output field intensity and power will change due to interaction of photons with bio molecules and variations in the above parameters can be observed. The fig. 4 represents the simulation results of a point defect which acts as a resonant cavity.

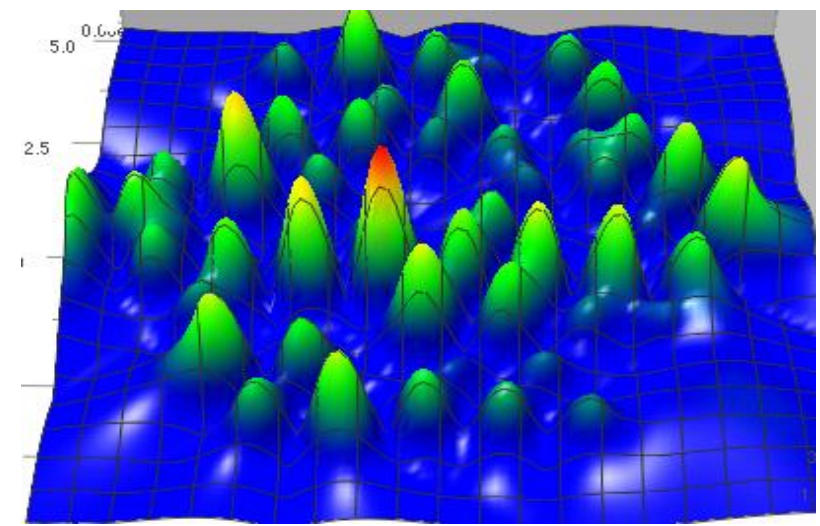

Fig. 4: A resonant cavity as a point defect

The electric field intensity variations for resonant cavity with and without biofluid are shown in table 1 . This application proves that the point defect can be used as biosensor.

Table 1: Comparison of magnitude of electric field intensity for a resonant cavity with and without biofluid

\begin{tabular}{lcc}
\hline Type of Structure & Magnitude of electric field intensity without biofluid & Magnitude of electric field intensity with biofluid \\
\hline Resonant cavity & 14.5 & 13.5 \\
\hline
\end{tabular}

\subsection{A $90^{\circ}$ bend waveguide}

A line defect in photonic crystal would guide light from one location to other. Once light is induced to travel along the waveguide, light travels into the bend without loss since the frequency of the guided mode lies within the PBG. Now if the biofluid is placed in the propagation path, the change in output field intensity and power can be observed. The fig. 5 shows the $90^{\circ}$ degree bend wave guide for a square lattice and the ratio of sphere radius to lattice constant is 0.3 .

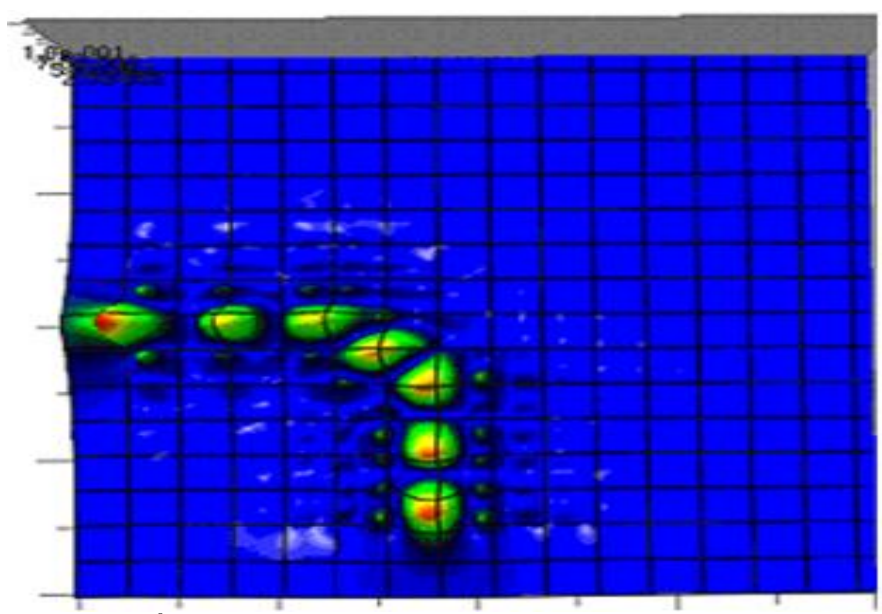

Fig. 5: $90^{\circ}$ Bend Waveguide with biofluid of refractive index $(n=1.37)$

The electric field intensity variations for $90^{\circ}$ bend waveguide with and without biofluid are shown in table 2 . This application proves that the line defect can be used as biosensor.

Table 2: Comparison of magnitude of electric field intensity for a waveguide with and without bio fluid

\begin{tabular}{lll}
\hline Type of Structure & Magnitude of electric field intensity without biofluid & Magnitude of electric field intensity with biofluid \\
\hline $90^{\circ}$ bend wave guide $(1000 \mathrm{THz})$ & 30.0 & 10.0 \\
\hline
\end{tabular}




\subsection{A Multi fluidic channel}

The defect channel couple light only when the appropriate refractive index material is present the channel. Without fluid flow, only the central channel is tuned to the input optical wavelength. The fig.6 represents the multi-fludic channel with the presence of biofluids of refractive indices $(1.37,1.4,1.0,2.0,1.35)$ and the light is guided in channels $1,2,3,4$ and 5 respectively.

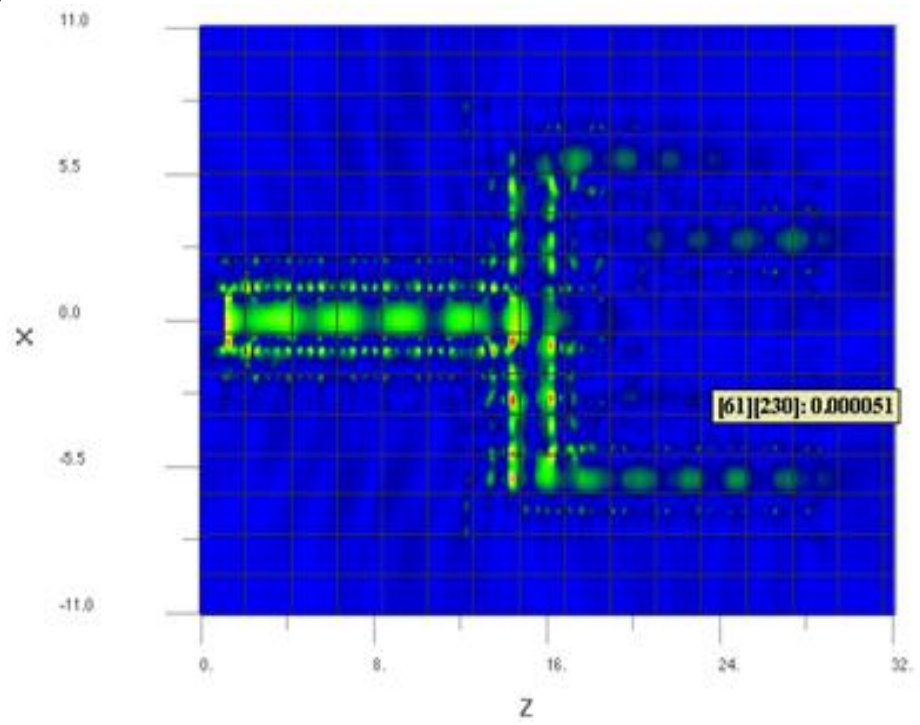

Fig. 6: Field distribution in the multi-fluidic channel

The power variations for multi-fluidic channel with biofluids where we can explore the sensitivity to small changes in the refractive index of the biofluid leads to large changes in power where this property can be used for sensing applications as shown in table 3 .

Table 3: Comparison of magnitude of electric field intensity for a multi-fluid channel for different biofluids

\begin{tabular}{|l|c|c|c|c|c|}
\hline Multi-fluid channel & $\mathrm{n}_{1}$ & $\mathrm{n}_{2}$ & $\mathrm{n}_{3}$ & $\mathrm{n}_{4}$ & $\mathrm{n}_{5}$ \\
\hline Refractive Index & 1.37 & 1.4 & 1.0 & 2.0 & 1.35 \\
\hline Magnitude of electric field intensity & 2.0 & 0.5 & 3.0 & 7.0 & 5.8 \\
\hline
\end{tabular}

The fig. 7 represents the electric field intensity as a function of frequency for a $90^{\circ}$ bend waveguide with and without biofluid where the extracted field intensity of waveguide with biofluid reflects intrinsic losses such as the absorption, and light scattering etc.

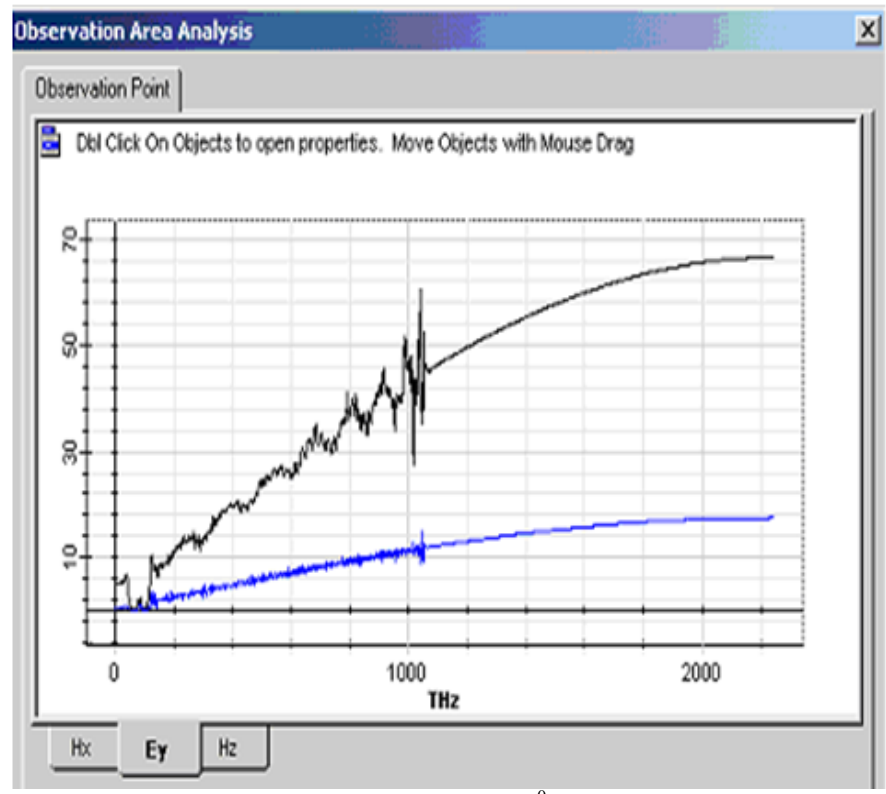

Fig: 7: Electric field intensity as function of frequency for $90^{\circ}$ bends waveguide with and without biofluid. 
Table 4: Power variations for $90^{\circ}$ bend waveguide with and without biofluid.

\begin{tabular}{|c|c|c|c|c|c|}
\hline $\begin{array}{l}\text { Mesh } \\
\text { points }\end{array}$ & $\begin{array}{l}\text { Power in } \mathrm{mW} / \mu \mathrm{m} \\
\text { without biofluid }\end{array}$ & $\begin{array}{l}\text { Power in } \mathrm{mW} / \mu \mathrm{m} \text { with } \\
\text { biofluid }(\mathrm{n}=1.33)\end{array}$ & $\begin{array}{l}\text { Power in } \mathrm{mW} / \mu \mathrm{m} \\
\text { with biofluid }(\mathrm{n}=1.37)\end{array}$ & $\begin{array}{l}\text { Power in } \mathrm{mW} / \mu \mathrm{m} \\
\text { with biofluid }(\mathrm{n}=1.465)\end{array}$ & $\begin{array}{l}\text { Power in } \mathrm{mW} / \mu \mathrm{m} \\
\text { with biofluid }(\mathrm{n}=1.6)\end{array}$ \\
\hline 20 & $3.251 * 10^{-12}$ & $8.054 * 10^{-14}$ & $5.835^{*} 10^{-13}$ & $1.002 * 10^{-12}$ & $1.351 * 10^{-13}$ \\
\hline 30 & $3.327 * 10^{-12}$ & $9.206 * 10^{-14}$ & $3.947 * 10^{-13}$ & $7.864 * 10^{-13}$ & $6.671 * 10^{-13}$ \\
\hline 40 & $3.672 * 10^{-12}$ & $2.021 * 10^{-14}$ & $5.377 * 10^{-13}$ & $7.164 * 10^{-13}$ & $6.796 * 10^{-13}$ \\
\hline 50 & $4.033 * 10^{-12}$ & $5.684 * 10^{-14}$ & $7.222 * 10^{-13}$ & $6.292 * 10^{-13}$ & $6.002 * 10^{-13}$ \\
\hline 60 & $4.254 * 10^{-12}$ & $3.943 * 10^{-14}$ & $6.066 * 10^{-13}$ & $6.164 * 10^{-13}$ & $4.263 * 10^{-13}$ \\
\hline 70 & $3.693 * 10^{-12}$ & $2.219 * 10^{-13}$ & $5.597 * 10^{-13}$ & $5.441 * 10^{-13}$ & $6.026 * 10^{-13}$ \\
\hline 79 & $2.740 * 10^{-12}$ & $3.384 * 10^{-13}$ & $8.937 * 10^{-13}$ & $4.403 * 10^{-13}$ & $6.214 * 10^{-13}$ \\
\hline 80 & $2.929 * 10^{-12}$ & $3.128 * 10^{-13}$ & $6.900 * 10^{-13}$ & $4.059 * 10^{-13}$ & $5.941 * 10^{-13}$ \\
\hline 90 & $1.753 * 10^{-12}$ & $3.031 * 10^{-14}$ & $7.537 * 10^{-13}$ & $2.308 * 10^{-13}$ & $6.211 * 10^{-13}$ \\
\hline
\end{tabular}

The biofluid comprises of certain analyte would lead to small shift in the refractive index of the biofluid due to absorption as shown in table 4 . This shift would in turn lead to large change in the power variations. As the index increases by 0.04 the change in power is observed and shifts systematically to higher value. Calculations show that for 0.04 variations in refractive index, the $86 \%$ change in power is observed.

\section{Conclusion}

Photonic crystals are scalable and hence they can be simulated at the micron level and later physically realized at the macroscopic level. An optimized simulation technique has to be met so that the efficiency at the macroscopic level should not be hindered. Further a compromise between the simulation and real time fabrication has to be made taking into consideration of the sensing device. The potential of photonic crystal devices is yet to be implored completely. Since signal flow is by light waves, high speed lab-on-chips can be realized which is highly desirable in the biophotonics arena for high speed sensing of biofluids.

\section{References}

[1] Steven G. Johnson and J. D. Joannopoulos, "Introduction to photonic crystals: Bloch's Theorem, band diagrams and gaps (But no defects)", MIT, February, 2003.

[2] Seunghyun Kim, Gregory P. Nordin, Jianhua Jiang and Jingbo Cai, "High efficiency $90^{\circ}$ silica waveguide bend using an air hole photonic crystal region", IEEE Photonics Technology Letters, vol. 16, no. 8, August 2004.

[3] M. Kamp, T. Happ, S. Mahnkopf, G. Duan, S. Anand and A. Forchel, "Semiconductor photonic crystals for optoelectronics", Proceedings of the eleventh International conference on modulated semiconductor structures, vol. 21, March 2004, pp. 802-808.

[4] J. D. Joannopoulos, Pierre R. Villeneuve and Shanhui Fan, "Photonic crystals: putting a new twist on light", MIT, vol. 386, March 1997, pp. 143-149.

[5] Masahiro Imada and Susumu Noda, "Recent progress of semiconductor photonic crystal", Proceedings of second IEEE conference on Nanotechnology, August 2002.

[6] Jamie R. Link and Micheal J. Sailor, "Smart dust: self-assembling, self orienting photonic crystals of porous Si", Proceedings of the National Academy of Sciences, USA, vol.100, no.19, 2000, pp. 10607-10610.

[7] Hua Xiao and Duanzheng Yao, "Analysis of the design of a new tunable photonic crystal filter at visible band", Physica E: Low-dimensional Systems and Nanostructures, vol. 27, Issues 1-2, March 2005, pp. 1-4.

[8] H. W. Tan and H. M. van Driel, "Nonlinear optical tuning of a two-dimensional silicon photonic crystal", Physical review B 70, 205110, 2004.

[9] B. Denecker, F. Olyslager, D. De Zutter, L. Klinkenbusch and L. Knockaert, "Efficient analysis of photonic crystal structures using a novel FDTD-technique", IEEE 2002, pp. 344-347.

[10] E. Ya. Glushko, "All-optical signal processing in photonic structures with nonlinearity", Optics communications, vol. 247, Issues 4-6, March 2005, pp. 275-280.

[11] Alexander Moroz, "Metallo-dielectric diamond and zinc-blende photonic crystals", The American physical society, Sep. 2002.

[12] John D. Joannopoulos, Steven G. Johnson, Joshua N. Winn and Robert D. Meade, "Photonic crystals: molding the flow of light", Second edition, Princeton University press, 2008.

[13] Allen Taflove and Susan C. Hagness, "Computational electrodynamics: the finite-difference time-domain method", Second edition Publishers - Artech House Boston, London 2000. 